\title{
Intravenous Remifentanil Analgaesia for an Obstetric Patient with Type I Neurofibromatosis and a Factor $V$ Leiden Mutation
}

José L. Gálvez,, "Carlos L. Errando, ${ }^{2,3}$ Silvia Serrano, ${ }^{4}$ Marga Martín-Ayuso, ${ }^{1}$ José M. Valverde-Mantecón ${ }^{1}$

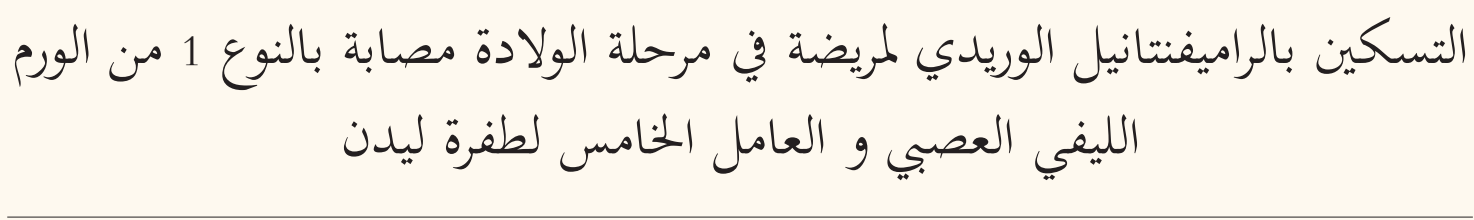

خوسيه لوس جالفيز، كارلوس لوس إراندو، سيلفيا سيرانو، مارجا مارتن-آيوسو، خوسيه ميجيول فالفيردي-مانتيكون

ABSTRACT: Type I neurofibromatosis is characterised by altered skin pigmentation and the growth of benign tumours, particularly along the peripheral nerves and central nervous system. We report a 36-year-old primigravida woman in labour who was admitted to the obstetric suite of the Hospital Sant Joan de Déu, Barcelona, Spain, in 2007 with hypothyroidism, type I neurofibromatosis and a factor V Leiden mutation. Due to a lack of cranial and spinal imaging data, an epidural was not indicated; instead, continuous intravenous remifentanil analgaesia was administered. The remifentanil infusion was self-titrated by the patient using a visual analogue scale, with the dosage ranging from 0.01 to $0.25 \mu \mathrm{g} / \mathrm{kg} /$ minute. Due to rotational dystocia, Kjelland-type forceps were used during the delivery. After birth, the infant was found to have Apgar scores of 9 and 10, with no maternal or neonatal adverse effects observed. Although still controversial, remifentanil may be a successful alternative for analgaesia in similar cases; however, the specific risks and benefits for each patient should be considered prior to administration.

Keywords: Obstetrical Analgesia; Drug Contraindications; Type 1 Neurofibromatosis; Factor V Deficiency; Case Report; Spain.

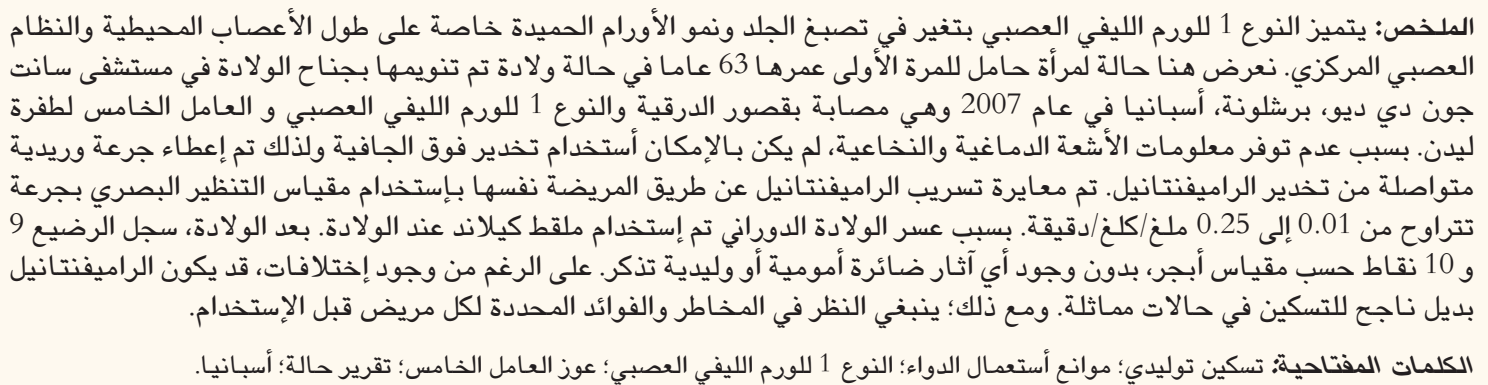

\section{$\mathrm{T}$} HE CONCURRENCE OF MULTIPLE GENETICALLY unrelated inherited diseases is rare and complicates analgaesic or anaesthetic management. Type I neurofibromatosis (NF) - also known as Von Recklinghausen's disease-is an autosomal dominant disease caused by a mutation in the NF1 gene on chromosome 17; it has a prevalence of 1 in 2,500-3,500 live births and accounts for $80 \%$ of all NF cases., ${ }^{1,2}$ Approximately half of all type I NF cases are inherited, while the rest are de novo mutations. ${ }^{2}$ The classical features of type 1 NF include benign tumours of the peripheral nerves (i.e. neurofibromas), pigmented milky-brown skin lesions, freckles in unexposed areas such as the armpits and iris hamartomas (i.e. Lisch nodules). ${ }^{3}$ While most patients are usually asympt- omatic, these features can result in compressive radiculopathies or neuropathies. Notably, the clinical presentation is quite variable even between affected members of the same family. ${ }^{2}$ Type I NF can be associated with first-trimester miscarriages and obstetric patients often require a Caesarean section delivery due to complications such as pelvic neurofibromas, pelvic bone abnormalities and kyphoscoliosis. ${ }^{3}$

Adults with type I NF can develop multiple cutaneous and subcutaneous neurofibromas which increase in number and size throughout their life. ${ }^{3}$ Plexiform neurofibromas can cause deformities, pain and functional problems and may sometimes become malignant. Osteopaenia, osteoporosis, bone overgrowth, short stature, macrocephalia, scoliosis, 
skeletal dysplasia (particularly of the sphenoid bone and vertebral wing) or pseudoarthrosis may also be present. ${ }^{3}$ Other alterations include arterial hypertension, vasculopathy, intracranial tumours, malignant peripheral nerve sheath tumours and, occasionally, seizures or hydrocephalus. Cognitive deficits and learning difficulties commonly occur in $30-45 \%$ of adults with NF, along with an increased risk of cancer. ${ }^{3}$

Most inherited hypercoagulable disorders are due to deficiencies of proteins $\mathrm{C}$ or $\mathrm{S}$ and antithrombin III, which occur in $15 \%$ of venous thrombosis cases. ${ }^{4}$ From a pathophysiological point of view, factor $\mathrm{V}$ Leiden (FVL) is the most common causative factor in this group of diseases. ${ }^{4}$ A FVL defect results from a mutation in the G1691A gene that encodes clotting factor V, which is inherited as an autosomal dominant trait; this leaves factor $\mathrm{V}$ resistant to inactivation by factor $\mathrm{V}$-activated protein C. ${ }^{4,5}$ In the normal population, up to $3-5 \%$ of people carry the FVL mutation. ${ }^{6}$ Pregnant women suffering from this deficiency appear to have an increased rate of spontaneous recurrent fetal loss. ${ }^{5}$ To the best of the authors' knowledge, no relationship between FVL deficiency and NF has yet been reported in the literature. Moreover, the occurrence of these conditions during pregnancy makes analgaesic management challenging during delivery. This report describes a pregnant woman who presented with both of these conditions and in need of analgaesia during labour.

\section{Case Report}

A 36-year-old primigravida woman at 39 gestational weeks and five days was admitted to the obstetric suite of the Hospital Sant Joan de Déu, Barcelona, Spain, in 2007 for the induction of labour. She weighed $84 \mathrm{~kg}$ and her height was $162 \mathrm{~cm}$. Her fetus was of low weight. She was known to have hypothyroidism, type I NF and a FVL mutation. These diseases were under medical control both before and during her pregnancy, with the hypothyroidism treated with levothyroxine. During the last trimester of her pregnancy, she had been prescribed 3,500 $\mathrm{U}$ of bemiparin per day; she had been advised to discontinue use 12 hours prior to delivery and/or the epidural puncture and to subsequently resume taking the drug 24-48 hours postpartum, provided there were no haemorrhagic complications.

Childbirth was induced using vaginal dinoprostone. After 24 hours, dilatation of the cervix was still unfavourable with a Bishop's score of $<6$. As such, oxytocin perfusion was initiated. A few hours later, amniorrhexis was performed and the patient requested analgaesia. Her coagulation profile showed a thromboplastin time of 12.6 seconds, $98 \%$ prothrombin activity, an activated partial thromboplastin time of 26.2 seconds, fibrinogen levels of $465 \mathrm{mg} / \mathrm{dL}$ and an international normalised ratio of 0.92. A clinical evaluation indicated that she had neurofibromas covering the entire surface of her back. Due to the lack of recent skull, central nervous system or spine imaging, neuraxial analgaesia could not be administered and alternative techniques for labour analgaesia were considered. Subsequently, a remifentanil infusion was agreed upon with the patient's consent. The potential adverse effects and complications of the drug were explained to the patient and a visual analogue scale (VAS) was used to continuously evaluate her pain.

During dilatation, the patient's blood pressure was monitored non-invasively every 10 minutes while her heart rate and pulse oximetry peripheral arterial oxygen saturation were monitored continuously. The fetal heart rate and uterine tone were also continuously recorded. A total of $150 \mathrm{mg}$ of ranitidine was administered via an intravenous injection, along with $2 \mathrm{~L} /$ minute of oxygen through nasal prongs. The patient was instructed on how to use and control the remifentanil infusion herself; however, the midwife and/or the anaesthesiologist assisted her with the infusion pump whenever necessary. The infusion was initiated at a rate of $0.01 \mu \mathrm{g} / \mathrm{kg} /$ minute with the maximum limit set at $0.25 \mu \mathrm{g} / \mathrm{kg} /$ minute. The patient's VAS scores increased from $0-3$ to $4-5$ by the time she was fully dilated towards the end of the labour, with the remifentanil infusion rate modified upon demand. The delivery was performed using Kjelland-type forceps due to rotational dystocia and an episiotomy was performed with local anaesthetic infiltration. After 395 minutes of labour, a male neonate weighing 3,120 g was born. His Apgar scores were 9 and 10 at one and five minutes, respectively. Cord blood tests were within the normal range and no haemodynamic alterations in the patient were observed, other than transient sinus tachycardia during the delivery.

\section{Discussion}

Remifentanil is an ultrashort-acting synthetic opioid with a short duration of action and no cumulative effects; its peak effect usually occurs 1-2 minutes after administration. ${ }^{7,8}$ It also has a very short half-life of approximately three minutes, independent of hepatic or renal function. ${ }^{8}$ An infusion rate of $0.1 \mu \mathrm{g} / \mathrm{kg} /$ minute preserves spontaneous breathing among patients who are awake. ${ }^{9}$ However, arterial hypotension, muscle stiffness, nausea and vomiting, chills, bradycardia, apnoea and respiratory depression have been reported 
as side-effects. ${ }^{7-10}$ Tolerance and hyperalgaesia have also been described. ${ }^{7,8}$

The concurrence of type I NF and FVL deficiency in a pregnant patient is an unusual challenge both from an obstetric point of view and in relation to the choice of analgaesia. Although the use of remifentanil is gradually becoming more common among obstetric patients, it is still a subject of some debate. ${ }^{-10}$ Moreover, remifentanil infusions are rarely reported among obstetric patients with comorbidities. In cases similar to the one described in this report, intramuscular or intravenous analgaesia with opiates (i.e. meperidine) or inhaled nitrous oxide are usually considered instead. ${ }^{10}$ However, previous research indicates that remifentanil is more effective than either of these options. ${ }^{7}$

In a multicentre study of pregnant women with FVL deficiency, Martinelli et al. noted a significant increase in the prevalence of venous thrombosis both during and after pregnancy. ${ }^{11}$ Although, anticoagulation with low-molecular-weight heparin (LMWH) would have permitted neuraxial analgaesia in the current case, the dose-response time frame in a patient with coexisting type I NF is still unclear., ${ }^{41,12}$ Harnett et al. have previously described the successful use of epidural analgaesia and LMWH in a pregnant woman with FVL deficiency. ${ }^{13}$ However, neuraxial imaging prior to invasive procedures is recommended for patients with type I NF and coexisting hereditary neuropathies, such as Charcot-Marie-Tooth disease. ${ }^{14}$ Since this was unavailable in the present case, the anaesthesiologists decided to forego neuraxial techniques despite the patient's normal creatinine clearance rate.

The occurrence of neurofibromas at the puncture site for the administration of anaesthesia or analgaesia can make the technical procedure either difficult or impossible. An epidural haematoma following a dural puncture has been reported in a parturient woman with NF. ${ }^{15}$ Spinal tumours have also been reported in $40 \%$ of patients with type I NF, even among asymptomatic cases; most of these tumours were lateral to the intervertebral foramen and would therefore have been at risk of trauma during a neuraxial puncture, especially if they had spread towards the midline. ${ }^{16}$ Another possible complication following a dural puncture is rapid decompression, particularly among patients with intracranial tumours; this could cause spinal cord injury or death as a result of the increased intracranial pressure. ${ }^{17}$ Moreover, regional neuraxial anaesthesia is usually contraindicated for patients with neurological symptoms.

Nevertheless, even intracranial space-occupying lesions do not always cause an increase in intracranial pressure; for example, individuals with no mass effect hydrocephalus or suggestive imaging findings have a minimal risk of herniation following a dural puncture. ${ }^{17}$ The parturient women at most risk include those who present with lesions that compress the brain tissue and cause midline deviation, either with or without obstruction of the cerebrospinal fluid flow. ${ }^{17}$ As such, cranial or neuraxial computed tomography or magnetic resonance imaging is highly recommended for pregnant women with both type I NF and FVL deficiency. ${ }^{12,17}$ In addition, it is also crucial to provide such patients with a comprehensive explanation of the associated risks and benefits of each analgaesic option.

\section{Conclusion}

The concurrence of rare diseases-such as type I NF and FVL deficiency-can complicate the management of analgaesia during labour, particularly with regards to standard neuraxial approaches. In the current case, the use of a usually contraindicated alternative, remifentanil, was successful; however, neuraxial analgaesia might also have been a feasible option, had recent imaging studies of the patient been available.

\section{References}

1. Vallejo MC. Anaesthesia recommendations for patients suffering from neurofibromatosis type 2. From: www.orpha. net/data/patho/Pro/en/NF2_EN.pdf Accessed: Jun 2017.

2. Friedman JM, Riccardi VM. Clinical epidemiological features. In: Friedman JM, Gutmann DH, MacCollin M, Riccardi VM, Eds. Neurofibromatosis: Phenotype, natural history, and pathogenesis, 3rd ed. Baltimore, Maryland, USA: Johns Hopkins University Press, 1999. Pp. 29-86.

3. Online Mendelian Inheritance in Man (OMIM). Entry \#162200: Neurofibromatosis, type I. From: http://omim.org/ entry/162200 Accessed Jun 2017.

4. Bande BD, Bande SB, Mohite S. The hypercoagulable states in anaesthesia and critical care. Indian J Anaesth 2014; 58:665-71. doi: 10.4103/0019-5049.144682.

5. Online Mendelian Inheritance in Man (OMIM). Entry \#612309: Coagulation factor V. From: http://omim.org/entry/612309 Accessed: Jun 2017

6. Leff J, Shore-Lesserson L, Fischer GW. Hematologic diseases. In: Fleisher LA, Ed. Anesthesia and Uncommon Diseases, 6th ed. Philadelphia, Pennsylvania, USA: Saunders, 2012. Pp. 350-68.

7. Blair JM, Dobson GT, Hill DA, McCracken GR, Fee JP. Patient controlled analgesia for labour: A comparison of remifentanil with pethidine. Anaesthesia 2005; 60:22-7. doi: 10.1111/j.13652044.2004.03975.x

8. Volmanen P, Sarvela J, Akural EI, Raudaskoski T, Korttila K, Alahuhta S. Intravenous remifentanil vs. epidural levobupivacaine with fentanyl for pain relief in early labour: A randomised, controlled, double-blinded study. Acta Anaesthesiol Scand 2008; 52:249-55. doi: 10.1111/j.1399-6576.2007. 01509.x.

9. Ohashi Y, Baghirzada L, Sumikura H, Balki M. Remifentanil for labor analgesia: A comprehensive review. J Anesth 2016; 30:1020-30. doi: 10.1007/s00540-016-2233-y. 
10. Kranke P, Girard T, Lavand'homme P, Melber A, Jokinen J, Muellenbach RM, et al. Must we press on until a young mother dies? Remifentanil patient controlled analgesia in labour may not be suited as a "poor man's epidural". BMC Pregnancy Childbirth 2013; 13:139. doi: 10.1186/1471-2393-13-139.

11. Martinelli I, Legnani C, Bucciarelli P, Grandone E, De Stefano V, Mannucci PM. Risk of pregnancy-related venous thrombosis in carriers of severe inherited thrombophilia. Thromb Haemost $2001 ; 86: 800-3$

12. Katz D, Beilin Y. Disorders of coagulation in pregnancy. Br J Anaesth 2015; 115:ii75-88. doi: 10.1093/bja/aev374.

13. Harnett MJ, Walsh ME, McElrath TF, Tsen LC. The use of central neuraxial techniques in parturients with factor V Leiden mutation. Anesth Analg 2005; 101:1821-3. doi: 10.1213/01. ANE.0000184135.00502.3E.
14. Kapur S, Kumar S, Eagland K. Anesthetic management of a parturient with neurofibromatosis 1 and Charcot-Marie Tooth disease. J Clin Anesth 2007; 19:405-6. doi: 10.1016/j. jclinane.2007.03.001

15. Esler MD, Durbridge J, Kirby S. Epidural haematoma after dural puncture in a parturient with neurofibromatosis. Br J Anaesth 2001; 87:932-4. doi: 10.1093/bja/87.6.932.

16. Thakkar SD, Feigen U, Mautner VF. Spinal tumours in neurofibromatosis type 1: An MRI study of frequency, multiplicity and variety. Neuroradiology 1999; 41:625-9. doi: $10.1007 / \mathrm{s} 002340050814$.

17. Leffert LR, Schwamm LH. Neuraxial anesthesia in parturients with intracranial pathology: A comprehensive review and reassessment of risk. Anesthesiology 2013; 3:703-18. doi: 10. 1097/ALN.0b013e31829374c2. 